Corresponding authors: nels2055@umn.edu; kathryn.wikenheiser-brokamp@ cchmc.org

(c) 2020 Oltvai et al. This article is distributed under the terms of the Creative Commons Attribution-NonCommercial License, which permits reuse and redistribution, except for commercial purposes, provided that the original author and source are credited.

Ontology terms: cyanosis; progressive pulmonary function impairment; respiratory insufficiency

Published by Cold Spring Harbor Laboratory Press

doi:10.1101/mcs.a005074

\section{Neonatal respiratory failure due to novel compound heterozygous mutations in the ABCA3 lipid transporter}

\author{
Zoltán N. Oltvai, ${ }^{1,2,7}$ Eric A. Smith, ${ }^{3,7}$ Katie Wiens, ${ }^{1}$ Lawrence M. Nogee, ${ }^{4}$ \\ Mark Luquette, ${ }^{1}$ Andrew C. Nelson, ${ }^{1,8}$ and Kathryn A. Wikenheiser-Brokamp ${ }^{5,6,8}$ \\ ${ }^{1}$ Department of Laboratory Medicine and Pathology, University of Minnesota, Minneapolis, Minnesota 55455, \\ USA; ${ }^{2}$ Department of Pathology and Laboratory Medicine, University of Rochester, Rochester, New York \\ 14642, USA; ${ }^{3}$ Medical Science Training Program, University of Cincinnati College of Medicine, Cincinnati, \\ Ohio 45219, USA; ${ }^{4}$ Department of Pediatrics, Johns Hopkins University, Baltimore, Maryland 21287, USA; \\ ${ }^{5}$ Division of Pathology and Laboratory Medicine and The Perinatal Institute, Division of Pulmonary Biology, \\ Cincinnati Children's Hospital Medical Center, Cincinnati, Ohio 45229, USA; ${ }^{6}$ Department of Pathology and \\ Laboratory Medicine, University of Cincinnati College of Medicine, Cincinnati, Ohio 45219, USA
}

Abstract The ATP-binding cassette transporter member A3 (ABCA3) is a lipid transporter with a critical function in pulmonary surfactant biogenesis. Biallelic loss-of-function mutations in $A B C A 3$ result in severe surfactant deficiency leading to neonatal respiratory failure with death in the first year of life. Herein, we describe a newborn with severe respiratory distress at birth progressing to respiratory failure requiring transplant. This patient was found to have a maternally inherited frameshift loss-of-function $A B C A 3$ mutation and a paternally inherited synonymous variant in $A B C A 3$ predicted to create a cryptic splice site. Additional studies showed reduced $A B C A 3$ expression in hyperplastic alveolar epithelial type II cells and lamellar body alterations characteristic of $A B C A 3$ deficiency, leading to a diagnosis of autosomal recessive ABCA3-related pulmonary surfactant dysfunction. This case highlights the need for an integrated, comprehensive approach for the diagnosis of inherited diseases when in silico modeling is utilized in the interpretation of key novel genetic mutations.

[Supplemental material is available for this article.]

\section{INTRODUCTION}

The ATP-binding cassette transporter member A3 (ABCA3) is a lipid transporter involved in pulmonary surfactant biogenesis. Biallelic loss-of-function mutations in the $A B C A 3$ gene result in severe surfactant deficiency leading to neonatal respiratory failure with death in the first year of life (Wambach et al. 2014a). Outcomes for infants and children with ABCA3 variants that cause uncertain disruption of protein function, such as missense, predicted novel splice sites, and in-frame insertions/deletions, are more challenging to predict, requiring complex clinical decision-making. Determining clinical significance of $A B C A 3$ mutations is further complicated by the fact that disease-associated mutations occur throughout the

\footnotetext{
${ }^{7}$ These authors contributed equally to this work.

${ }^{8} \mathrm{Co}$-senior authors.
} 
gene and are typically unique to each affected proband or pedigree (Wambach et al. 2014a; Peca et al. 2015; Schindlbeck et al. 2018). As utilization of multigene genetic testing increases in clinical practice, identification of variants of unknown clinical significance is becoming more frequent and can contribute to diagnostic confusion rather than clarity. Thus, reporting novel variants and patient outcomes is critical for defining the significance of rare genetic variants.

In this report, we describe a newborn with severe respiratory distress at birth progressing to respiratory failure requiring transplant who was found to have novel compound heterozygous $A B C A 3$ variants: a maternally inherited frameshift mutation and a paternally inherited synonymous variant predicted to create a cryptic splice site. Lung biopsy prior to transplantation showed chronic pneumonitis of infancy, supporting a surfactant dysfunction disorder and prompting genetic analysis. Weak staining for $A B C A 3$ was detected in the hyperplastic alveolar epithelial type II cells (AEC2) by immunohistochemical analysis. Lamellar body alterations characteristic of ABCA3 deficiency were identified by ultrastructural analysis. Together the patient's clinical findings, lung pathology, and genetic results confirmed a diagnosis of autosomal recessive ABCA3-related pulmonary surfactant dysfunction for this patient. This case identifies a novel potential aberrant splicing variant for ABCA3-related neonatal respiratory failure and highlights the need for an integrated, comprehensive approach for diagnosis of inherited diseases when interpretation of key novel genetic mutations is based on in silico modeling.

\section{RESULTS}

\section{Case Presentation}

The patient was the first-born male child of nonconsanguineous parents. He was born full term without complications by cesarean section delivery with Apgar scores of 8-9. Shortly after birth, the newborn started grunting and became dusky with slight tachycardia. A diagnosis of respiratory distress was made, and continuous positive airway pressure (CPAP) was started. His color improved despite continued grunting with subcostal retractions and decreasing Apgar score of 7 . Lungs were clear on examination, and thrice suctioning revealed thick clear secretions. CPAP continued for 20 min with high-flow nasal cannula (HFNC) oxygen therapy. Initial oxyhemoglobin saturation was $98 \%-100 \%$, which gradually declined to $91 \%-93 \%$, resulting in the patient being transferred to the neonatal intensive care unit. A broad differential diagnosis was considered, leading to a comprehensive evaluation including chest radiograph, echocardiogram, sepsis workup, head ultrasound, and renal ultrasound. The cardiac echography identified a small patent foramen ovale, and renal ultrasound showed moderate hydronephrosis of the left kidney. Chest radiograph and studies for an infectious etiology were negative, including bacterial cultures (performed on lung tissue biopsy, tracheal aspirates, blood, urine, and cerebrospinal fluid [CSF]); acid-fast bacteria (AFB), fungal, and viral cultures on lung tissue; Neisseria meningitides, Escherichia coli, and Group B strep antigen on CSF; enteroviral polymerase chain reaction (PCR), herpes simplex virus (HSV) PCR, and respiratory viral PCR panel on nares swab; adenoviral, enteric bacterial, and cryptosporidium antigen on stool; and viral nucleic acid amplification testing on stool. A three-generation pedigree was obtained and was noncontributory for lung disease.

Despite being treated with several doses of surfactant and multiple courses of methylprednisolone for clinical suspicion of surfactant deficiency, the patient remained critically ill in respiratory distress. The lack of response to exogenous surfactant and low suspicion for infection led to congenital surfactant metabolism and/or pulmonary fibrosis-related disorders being considered as etiologies for his clinical presentation. Lung wedge biopsy was 
obtained to define the disease process. Peripheral blood samples were also obtained from the infant and parents for genetic analysis.

\section{Lung Pathology}

The lung biopsy had key histologic features of chronic pneumonitis of infancy (Fig. 1). Alveolar septa were thickened by mesenchymal cells and hyperplastic AEC2 with minimal inflammation and abundant intra-alveolar macrophages admixed with patchy accumulations of proteinaceous material. These morphologic features raised strong suspicion for a surfactant dysfunction disorder including genetic mutations in SP-B (SFTPB), SP-C (SFTPC), and ABCA3 (ABCA3).
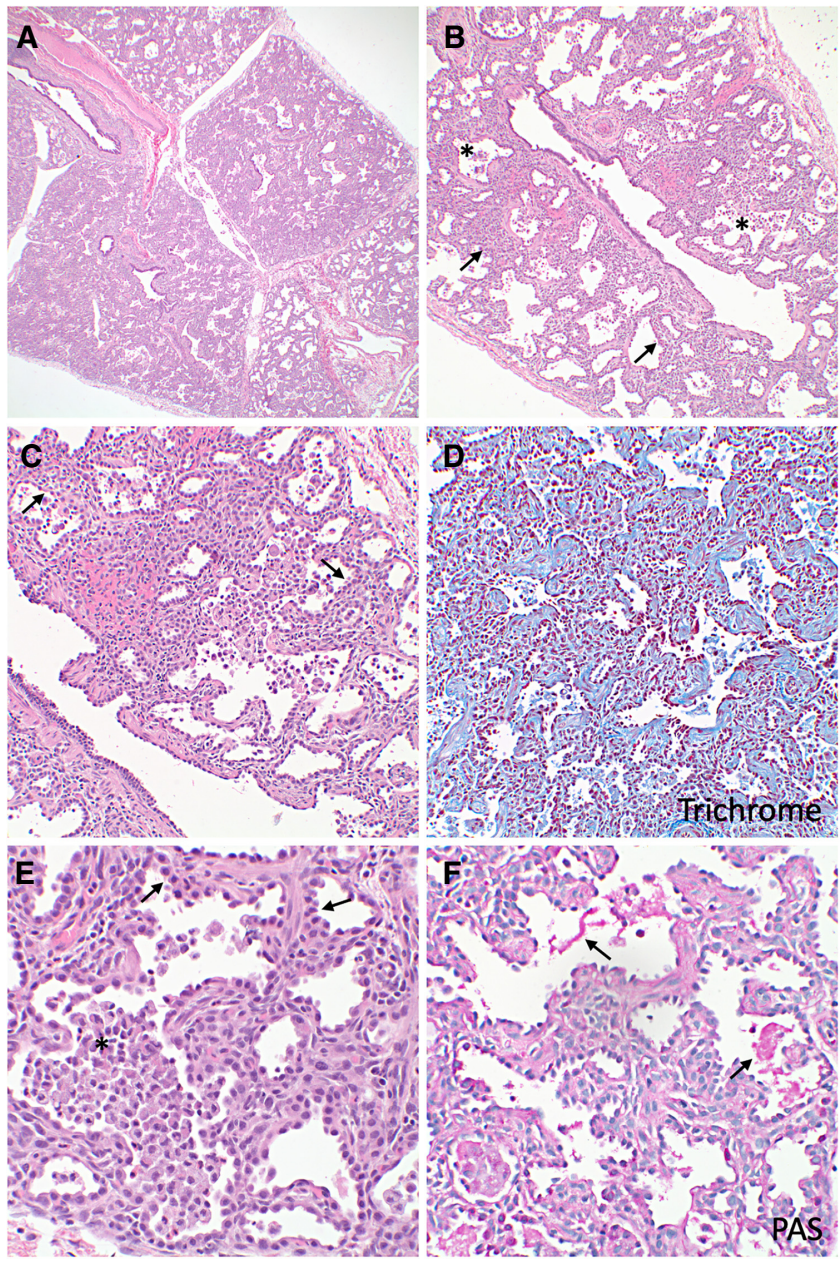

Figure 1. Lung pathology showing chronic pneumonitis of infancy pattern. The overall lobular architecture of the lung is preserved $(A)$ with a combination of interstitial $\left(B\right.$, arrows) and airspace $\left(B,{ }^{*}\right)$ abnormalities including some lobular remodeling $(C)$. The alveolar septa are thickened $(C$, arrows) by mesenchymal cells, minimal chronic inflammation, some interstitial fibrosis ( $D$, blue by trichrome stain), and diffuse alveolar epithelial pneumocyte hyperplasia ( $E$, arrows). Intra-alveolar macrophages, many with foamy cytoplasm, are abundant $\left(E,{ }^{*}\right)$ with small patchy accumulations of periodic acid-Schiff (PAS) stain-positive proteinaceous material $(F$, arrows, PAS stain). Hematoxylin and eosin (H\&E)-stained images are shown except where alternate stain is indicated. Original magnifications, $40 \times(A) ; 100 \times(B) ; 200 \times(C, D) ; 400 \times(E, F)$. 
Immunohistochemical studies on the lung biopsy showed weak AEC2 staining for ABCA3 that was markedly decreased compared to a control pediatric nontransplanted donor lung (Fig. 2A). In contrast, strong AEC2 immunostaining was seen with antibodies against the SP-B mature and proprotein (proSP-B) (Fig. 2C,D). Moderate staining for the SP-C proprotein (proSP-C) was present, being only slightly reduced compared to the control lung (Fig. 2B). Importantly, extracellular staining for proSP-C, which is associated with its misprocessing in the context of SP-B deficiency, was absent (Fig. 2B; Nogee et al. 2000). Together, the markedly reduced $A B C A 3$ protein expression, strong proSP-B, and SP-B protein staining and lack of proSP-C accumulation in the alveolar spaces supported an ABCA3 abnormality, as described previously (Brasch et al. 2006).

ABCA3 deficiency results in characteristic changes in lamellar bodies (LBs), the surfactant-storing organelles in AEC2. Ultrastructural analysis in this case revealed numerous pleomorphic cytoplasmic inclusions in AEC2 with aggregates of electron-dense material and absence of concentric lamellae characteristic of normal mature LBs (Fig. 2E,F). AEC2s containing more normal-appearing LBs were also identified, but these cells also contained abnormal electron-dense aggregates (Fig. 2G,H). The combination of the patient's clinical findings, lung morphology, and immunostaining results (obtained within 3 wk from birth) and ultrastructural findings (obtained $5 \mathrm{wk}$ from birth) supported a diagnosis of ABCA3-related pulmonary surfactant dysfunction disorder.

\section{Sequencing Results}

Concomitantly with the lung biopsy analyses, next-generation sequencing (NGS) was performed on a peripheral blood sample from the patient using a Clinical Laboratory Improvement Amendments (CLIA)-validated, laboratory-developed NGS platform (Hartman et al. 2019). The custom gene panel included genes associated with surfactant metabolism dysfunction, pulmonary fibrosis, and neonatal respiratory distress (ABCA3, CSF2RA, CSF2RB, NKX2-1, SFTPA2, SFTPB, SFTPC, SFTPD, PARN, RTEL1, TERT, and TOLLIP).

Two variants in the ABCA3 gene (NM_001089.2) were identified (preliminarily results reported 5 wk after birth with final report issued 2 wk later): a heterozygous frameshift mutation c.4885_4886insG, p.Ala1629GlyfsX15 and a heterozygous synonymous variant c.2883C > T, p.Gly961Gly (rs1298655924) (Table 1; Supplemental Fig. 1). No other sequence variants or copy-number alterations were detected in the tested genes. Subsequently, the ABCA3 variants were determined to be in trans in the proband: The unaffected mother had the heterozygous frameshift mutation c.4885_4886insG, p.Ala1629GlyfsX15, whereas the unaffected father had the heterozygous c.2883C > T, p.Gly961Gly variant (Supplemental Fig. 2).

The c.4885_4886insG (p.Ala1629GlyfsX15) frameshift variant is located in exon 31 of the total 33-exon ABCA3 gene and is predicted to result in a premature stop codon 15 amino acids into the shifted reading frame. Although this specific variant is novel, other predicted loss-of-function $A B C A 3$ variants both upstream of and downstream from this variant have been reported as disease-causing. This variant is also absent from the gnomAD population database of more than 140,000 controls (Karczewski et al. 2019). Based on this evidence, the novel variant was interpreted as likely pathogenic per American College of Medical Genetics (ACMG) guidelines (Richards et al. 2015).

The c.2883C > T (p.Gly961Gly) ABCA3 novel variant is synonymous and does not occur at an intron/exon boundary. However, it is absent from the gnomAD population database and two splice site algorithms (NetGene2 and NNSplice [a.k.a. Fruitly]) consistently predicted creation of a novel cryptic donor splice site within exon 21 of the gene that may lead to aberrant splicing of the ABCA3 pre-mRNA (Supplemental Fig. 3). Given the absence of the c. $2883 \mathrm{C}>\mathrm{T}$ variant in control populations, the consistent in silico prediction of a deleterious 


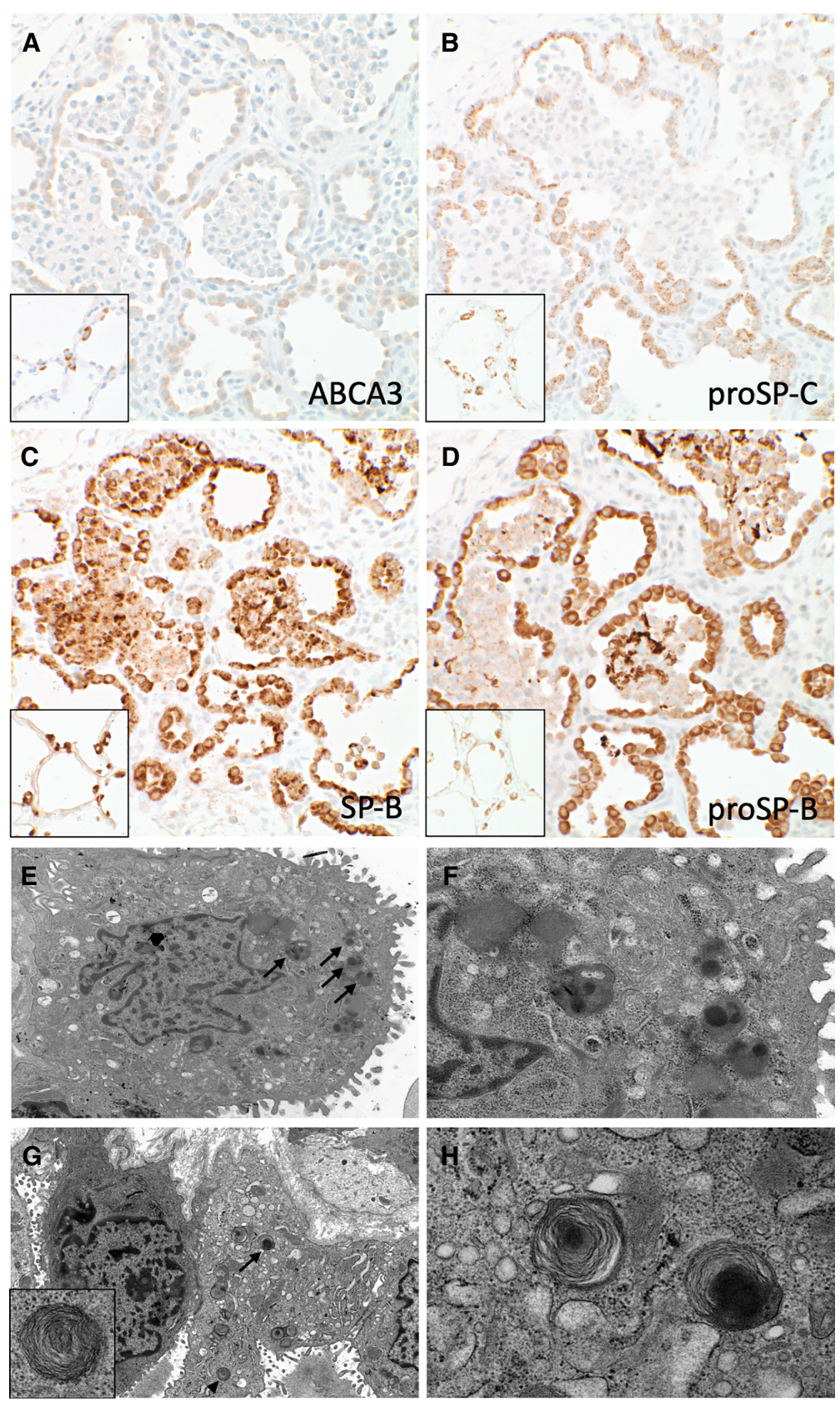

Figure 2. Lung has reduced $A B C A 3$ expression and abnormal lamellar bodies with retained expression of surfactant proteins SPB and SPC. Alveolar spaces are lined by type II epithelial cells with weak immunostaining for $\mathrm{ABCA} 3(\mathrm{~A}$, brown) and strong staining with antibodies generated against the SP-C proprotein ( $B$, brown), SP-B mature protein ( $C$, brown), and SP-B proprotein ( $D$, brown). Insets show staining for the respective proteins in a control nontransplanted pediatric donor lung. Note similar immunostaining intensities between the control and patient lung for all proteins except $A B C A 3$, which has markedly reduced staining in the patient lung compared to the control lung. Numerous pleomorphic cytoplasmic inclusions are present in alveolar type ll cells by electron microscopic analysis ( $E$, arrows). The inclusions contain aggregates of electron-dense material and lack the concentric lamellae characteristic of normal lamellar bodies $(F)$. Cells containing both normal $(G$, arrowhead and inset) and abnormal lamellar bodies $(G$, arrow) with tightly packed concentric membranes and aggregates of electron dense material $(H)$ were present. Original magnifications, $400 \times(A-D) ; 4000 \times(E)$; $10,000 \times(F) ; 2500 \times(G) ; 25,000 \times(G$, inset); $15,000 \times(H)$. 


\begin{tabular}{|c|c|c|c|c|c|c|c|c|}
\hline Gene & Chromosome & $\begin{array}{l}\text { HGVS DNA } \\
\text { reference }\end{array}$ & $\begin{array}{l}\text { HGVS protein } \\
\text { reference }\end{array}$ & Variant type & $\begin{array}{c}\text { Predicted effect } \\
\text { (substitution, deletion, } \\
\text { etc.) }\end{array}$ & $\begin{array}{c}\mathrm{dbSNP} / \mathrm{db} V a r \\
\text { ID }\end{array}$ & $\begin{array}{c}\text { Genotype } \\
\text { (heterozygous/ } \\
\text { homozygous) }\end{array}$ & $\begin{array}{l}\text { Parent of } \\
\text { origin }\end{array}$ \\
\hline$A B C A 3$ & $\begin{array}{c}\text { Chr 16:g.2338148G > } \\
\text { A GRCh37 (hg19) }\end{array}$ & $\begin{array}{r}\text { NM_001089.2 } \\
\text { c.2883C }>T\end{array}$ & $\begin{array}{r}\text { p.Gly961Gly } \\
\text { (exon 21) }\end{array}$ & Synonymous & $\begin{array}{l}\text { Cryptic splice } \\
\text { site activation }\end{array}$ & rs1298655924 & Heterozygous & Paternal \\
\hline ABCA3 & $\begin{array}{c}\text { Chr 16:g.2327903_ } \\
\text { 2327904insC } \\
\text { GRCh37 (hg19) }\end{array}$ & $\begin{array}{c}\text { NM_001089.2 } \\
\text { c.4885dup }\end{array}$ & $\begin{array}{l}\text { p.Ala1629GlyfsX15 } \\
\text { (exon 31) }\end{array}$ & $\begin{array}{l}\text { Frameshift } \\
\text { insertion }\end{array}$ & $\begin{array}{l}\text { Frameshift with } \\
\text { premature } \\
\text { truncation }\end{array}$ & N/A & Heterozygous & Maternal \\
\hline
\end{tabular}

splicing effect, its presence in trans with a pathogenic variant, and the histopathologic assessment consistent with surfactant metabolism dysfunction, this variant was also classified as likely pathogenic per ACMG guidelines (Richards et al. 2015). Taken together, the patient's typical clinical phenotype, ABCA3 genetic alterations (including variant segregation studies), and lung histologic, immunohistochemical, and ultrastructural pathology resulted in a diagnosis of autosomal recessive ABCA3-related pulmonary surfactant dysfunction disorder.

\section{Post-Transplant Clinical Course}

The patient underwent successful bilateral lung transplant 15 wk after birth. The gross lung weights were elevated at 186 and $141 \mathrm{~g}$ for right and left lungs, respectively. The explanted lungs had widespread histopathologic features of chronic pneumonitis of infancy, similar to the diagnostic biopsy. The left upper lobe had superimposed acute bronchopneumonia and prominent congestion. The patient's post-transplant course has remained free of infectious or rejection complications to date. Serial transbronchial biopsies in the 9-mo period post-lung transplant have been negative for acute and chronic rejection, and bronchoalveolar lavage samples have been variably cellular with a preponderance of intra-alveolar macrophages.

\section{DISCUSSION}

Mammalian breathing is dependent on the extensive gas exchange surface area provided by expanded lung alveoli with patency maintained by lipid-rich surfactant at the air-liquid interface. Surfactant function is dependent on proper synthesis, transport, and secretion by AEC2, as well as uptake and recycling by AEC2 and alveolar macrophages. Quantitative or qualitative changes in surfactant can result in alveolar collapse. Likewise, accumulation of surfactant in the alveolar airspaces, as occurs in pulmonary alveolar proteinosis, impedes gas exchange (Perez-Gil and Weaver 2010).

The major components of pulmonary surfactant are phospholipids ( $80 \%)$, neutral lipids (mainly cholesterol, $\sim 10 \%)$, and two hydrophobic peptides (1\%-2\%): surfactant protein B (SP-B) and surfactant protein C (SP-C) (Perez-Gil and Weaver 2010). AEC2s synthesize and assemble the lipid and protein components into complexes that are stored as tightly packed membranes in specialized organelles_the LBs—until they are secreted into the alveolar airspaces.

$A B C A 3$ is required for transport and organization of surfactant phospholipids in LBs (Perez-Gil and Weaver 2010). ABCA3 deficiency results in the replacement of mature LBs by structures containing densely packed concentric rings with electron-dense inclusions 
forming a characteristic "fried-egg" appearance (Edwards et al. 2005; Bruder et al. 2007; Doan et al. 2008). In 16 human infants with severe neonatal surfactant metabolism dysfunction, Shulenin et al. (2004) identified 12 different homozygous or compound heterozygous mutations in the $A B C A 3$ gene associated with $A B C A 3$ protein deficiency (Shulenin et al. 2004). Severely diminished $A B C A 3$ function alters transport of the major surfactant phospholipids-phosphatidylglycerol and phosphatidylcholine-decreasing quantity and quality of surfactant at the air-liquid interface (Garmany et al. 2006). Accumulation of this dysfunctional surfactant results in reduced alveolar macrophage activity, compromised gas exchange, and secondary lung pathologies. A cause-effect relationship between $A B C A 3$ deficiency and abnormal LB biogenesis was confirmed by Abca3 disruption in murine models that led to neonatal lethal respiratory distress syndrome (RDS) associated with loss of mature LBs and decreased phospholipid production (Ban et al. 2007; Cheong et al. 2007; Fitzgerald et al. 2007; Hammel et al. 2007). In the present case, markedly reduced ABCA3 immunostaining and the characteristic abnormal LBs in AEC2 by electron microscopy were critical for classifying a novel genetic mutation as pathogenic, demonstrating the diagnostic importance of protein expression studies and ultrastructural analysis in evaluating neonatal respiratory distress.

ABCA3 function is mediated by two six-unit transmembrane structures that form a channel for ATP-dependent transport of lipids into LB (Fig. 3A; Paolini et al. 2015). The mutations

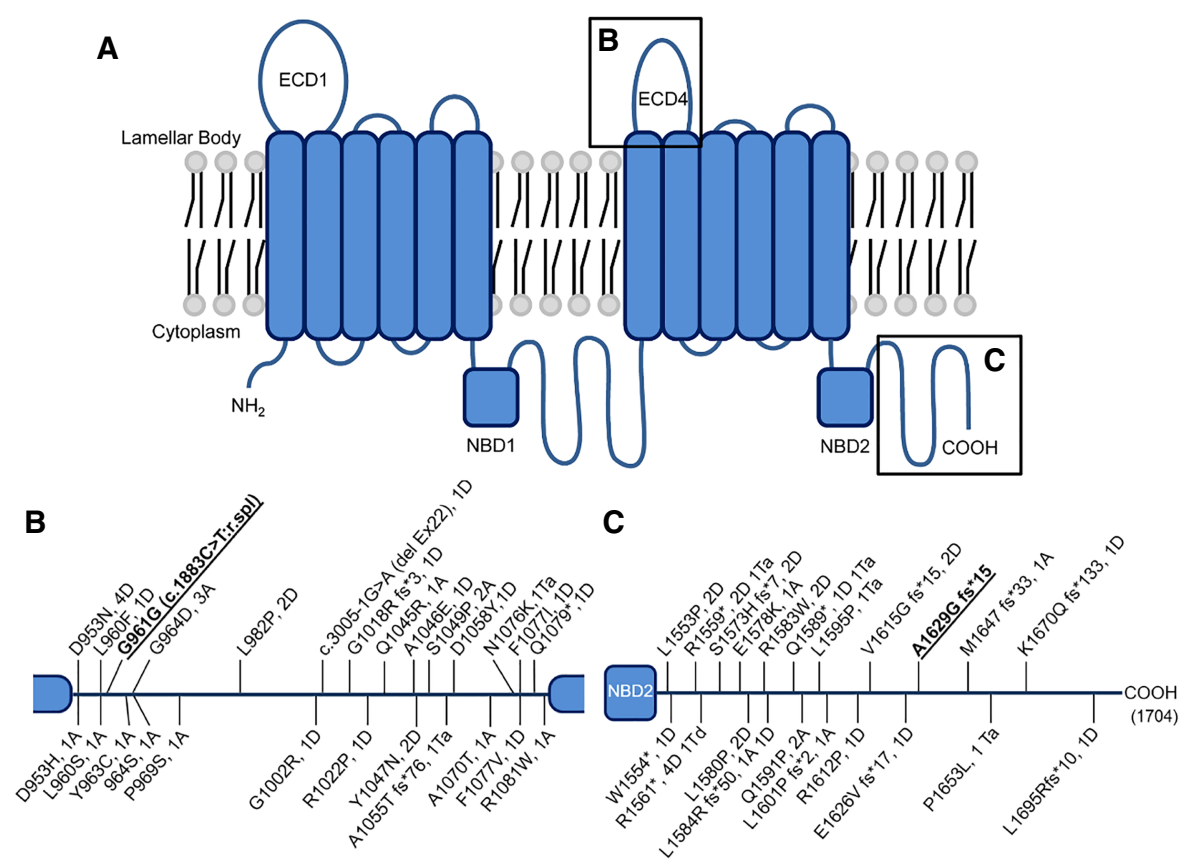

Figure 3. Known mutations in the ECD4 and carboxy-terminal domains of $A B C A 3$. (A) The ABCA3 protein has two six-unit transmembrane structures each paired with a nucleotide-binding domain (NBD). Between each transmembrane unit are "extracellular domains" (ECDs) within the inner compartment of the lamellar body, and intracellular domains in the cytoplasm. The mutations identified in this report are in ECD4 (B) and the carboxy-terminal region $(C)$. (B) Mutations in the ECD4 domain are represented as amino acid substitutions, cDNA nucleotide changes resulting in alternative splicing arising from synonymous mutations, and cDNA nucleotide changes resulting in large exon deletions. Following each mutation is the number of reported cases and clinical outcome: (A) alive, (D) died, (Ta) alive post-transplant, (Td) died post-transplant. The novel mutation in this case is bold and underlined. $(C)$ Carboxy-terminal domain mutations are represented as in $B$. Additional clinical information and references for individual mutations are in Supplemental Tables 1 and 2. 
identified in the current patient are located in the ECD4 and carboxy-terminal domains, a frequent location for mutations in ABCA3-deficiency-related disease (Fig. 3B,C; Supplemental Table 1). Within these specific domains, missense mutations reported in clinical cases lead to diminished ATPase activity of ABCA3 or prevent ABCA3 trafficking from the endoplasmic reticulum to the LB (Matsumura et al. 2006). Comparatively, frameshift mutations in the highly conserved carboxy-terminal domain likely abrogate both the LB localization and ATPase activity. It is expected that the p.Ala1629GlyfsX15 mutation in this case also disrupts one or both of these functions as three unique downstream frameshift mutations have proven fatal (Fig. 3C; Wambach et al. 2014b; Akil and Fischer 2018; El Boustany et al. 2018).

Because $A B C A 3$ deficiency is an autosomal recessive disorder, we propose that the novel c.2883C > T p.Gly961Gly donor splice site variant further reduces the number of normal ABCA3 transcripts to a symptomatic level in this patient (consistent with the very weak ABCA3 immunostaining; Fig. 2A). To our knowledge, this is the first report of a cryptic, exonic splicing variant in $A B C A 3$, despite synonymous mutations being identified in more than 330 patients with neonatal RDS (Wambach et al. 2014b). In the reported cases, algorithms did not predict disruption to RNA splicing, and no clear mechanism for RDS was identified (Wambach et al. 2014b). The progression of the current patient to respiratory failure requiring transplant combined with the very weak $A B C A 3$ immunostaining provides evidence that the p.Gly961Gly mutation behaves as a "mosaic null" allele, potentially with weak residual $A B C A 3$ expression due to variable usage of the wild-type rather than novel mutation-created splice site. Preferential usage of the mutation-created cryptic splice site would be similar to a nonsense or frameshift mutation, highlighting a novel mechanism for ABCA3 deficiency. An appropriate clinical sample for RNA analysis was not available to confirm this predicted mechanism; therefore, future studies are necessary to directly demonstrate the impact of this variant on $A B C A 3$ splicing and expression.

Reaching a final diagnosis in this case was crucially dependent on integration of clinical, pathologic, and genetic findings. The clinical presentation and lung pathology on biopsy identified a surfactant dysfunction disorder as a prime etiologic consideration. Although immunohistochemical staining cannot definitively identify the causative gene abnormality, it provided valuable support for a surfactant dysfunction disorder with a quick turnaround time to guide clinical decision-making. Electron microscopy can provide a specific diagnosis and should be included in the evaluation of lung biopsies from infants with respiratory distress. Concurrent NGS identified the genetic basis for the disease with in silico functional predictions for the novel $A B C A 3$ variant being critical to establishing the diagnosis. It is important to note that use of increasingly available rapid exome/genome sequencing (Meng et al. 2017; Clark et al. 2019) could further accelerate the establishment of timely molecular diagnosis in newborns with rapidly deteriorating pulmonary function. In our case, a rapid genetic diagnosis would have supported the diagnosis of ABCA3-related surfactant deficiency earlier in the patient's course; however, integration of all morphologic, laboratory, and clinical information is still necessary to arrive at a final diagnostic conclusion when genetic analysis reveals a novel mutation and genetic mechanism as illustrated by the current case.

\section{METHODS}

\section{Immunohistochemistry}

Immunohistochemistry was performed with the Roche Ventana BenchMark Ultra System with Tris-EDTA (ABCA3) or citrate (proSP-C, SP-B, proSP-B) antigen retrieval using the following antibodies from Seven Hills Bioreagents at the indicated dilutions: $A B C A 3$ (WRAB-ABCA3; 
COLD SPRING HARBOR Molecular Case Studies
A newborn with novel $\mathrm{ABCA} 3$ mutation
1:200); pro-SP-C (WRAB-9337; 1:2000); mature SP-B (WRAB-48604; 1:2000); and proSP-B (WRAB-55522; 1:800).

\section{Genomic Sequencing}

NGS was performed on a peripheral blood sample using a CLIA-validated, laboratory-developed NGS platform, as previously described (Hartman et al. 2019). Briefly, genomic DNA was extracted from a patient peripheral blood sample and quantified using the Qubit 2.0 fluorometer (ThermoFisher Scientific). Library preparation was carried out by tagmentation following Nextera protocols (Nextera XT library preparation; Illumina, Inc.). Target enrichment was performed by hybrid capture using vendor-customized baits (Integrated DNA Technologies) and Illumina Rapid capture reagents per the manufacturer's protocol. NGS was performed using Illumina NovaSeq instrument (version 3 chemistry, 300-base pair [bp] paired-end reads; Illumina, Inc.). FASTQ files were processed through a custom designed bioinformatics pipeline, Scanlndel (Yang et al. 2015). Average coverage by base was $140 x$, with $100 \%$ of all bases in the clinically reported genes achieving minimum $15 x$ coverage (see Supplemental Table 3). Variant call files (vcfs) were filtered to remove subthreshold calls with $<20 \times$ coverage and/or variant allele frequency (VAF) less than defined. Clinically relevant mutations from this VAF were annotated by a board-certified molecular genetic pathologist with Genelnsight software (Sunquest) and reported. Parental blood samples were processed as above and tested for the identified ABCA3 variants using targeted Sanger sequencing.

\section{ADDITIONAL INFORMATION}

\section{Data Deposition and Access}

The consent documentation signed and verbally acknowledged by the patient's family does not expressly allow submission of full sequencing data (FASTQ, BAM/BAI, VCF) for the germline clinical NGS capture to external data repositories (see below). The variants were deposited in ClinVar (https://www.ncbi.nlm.nih.gov/clinvar/) and can be found under accession numbers SCV001334048.1 and SCV001334049.1.

\section{Ethics Statement}

The patient's parent signed the institution-approved, standard consent for clinical diagnostic testing by NGS, including agreement to the opt in/opt out clause for use of genetic information for research purposes. Further verbal consent from the parent was obtained and documented in the electronic medical record by a clinical care team member (genetic counselor) to publish nonidentifying details about the patient's specific diagnosis, clinical test results, and care for advancement of clinical-academic practice. Neither of these consent mechanisms allow for sharing of genetic information beyond that clinically relevant and reported in the manuscript.

\section{Acknowledgments}

We thank the family for allowing us to publish their case. The control nontransplanted donor lung was obtained from LifeCenter Organ Donor Network (Cincinnati, $\mathrm{OH}$ ) in collaboration with Francis X. McCormack, MD (University of Cincinnati, Cincinnati, OH). This study was also supported by services from the Pathology Research Core shared facility in the Cincinnati Children's Research Foundation with specific acknowledgment of the assistance of Robin Sams.
Received December 12, 2019; accepted in revised form March 3, 2020.
Competing Interest Statement

The authors have declared no competing interest.

Oltvai et al. 2020 Cold Spring Harb Mol Case Stud 6: a005074 


\section{Author Contributions}

Z.N.O., E.A.S., K.W., L.M.N., A.C.N., and K.A.W.-B. wrote the manuscript with input from M.L. All authors have read and approved the final version of the manuscript.

\section{REFERENCES}

Akil N, Fischer AJ. 2018. Surfactant deficiency syndrome in an infant with a C-terminal frame shift in ABCA3: a case report. Pediatr Pulmonol 53: e12-e14. doi:10.1002/ppul.23994

Ban N, Matsumura Y, Sakai H, Takanezawa Y, Sasaki M, Arai H, Inagaki N. 2007. ABCA3 as a lipid transporter in pulmonary surfactant biogenesis. J Biol Chem 282: 9628-9634. doi:10.1074/jbc.M611767200

Brasch F, Schimanski S, Muhlfeld C, Barlage S, Langmann T, Aslanidis C, Boettcher A, Dada A, Schroten H, Mildenberger $E$, et al. 2006. Alteration of the pulmonary surfactant system in full-term infants with hereditary ABCA3 deficiency. Am J Respir Crit Care Med 174: 571-580. doi:10.1164/rccm.200509-1535OC

Bruder E, Hofmeister J, Aslanidis C, Hammer J, Bubendorf L, Schmitz G, Rufle A, Buhrer C. 2007. Ultrastructural and molecular analysis in fatal neonatal interstitial pneumonia caused by a novel $A B C A 3$ mutation. Mod Pathol 20: 1009-1018. doi:10.1038/modpathol.3800928

Cheong N, Zhang H, Madesh M, Zhao M, Yu K, Dodia C, Fisher AB, Savani RC, Shuman H. 2007. ABCA3 is critical for lamellar body biogenesis in vivo. J Biol Chem 282: 23811-23817. doi:10.1074/jbc.M703927200

Clark MM, Hildreth A, Batalov S, Ding Y, Chowdhury S, Watkins K, Ellsworth K, Camp B, Kint Cl, Yacoubian C, et al. 2019. Diagnosis of genetic diseases in seriously ill children by rapid whole-genome sequencing and automated phenotyping and interpretation. Sci Transl Med 11: eaat6177. doi:10.1126/scitranslmed.aat6177

Doan ML, Guillerman RP, Dishop MK, Nogee LM, Langston C, Mallory GB, Sockrider MM, Fan LL. 2008. Clinical, radiological and pathological features of ABCA3 mutations in children. Thorax 63: 366-373. doi:10.1136/thx.2007.083766

Edwards V, Cutz E, Viero S, Moore AM, Nogee L. 2005. Ultrastructure of lamellar bodies in congenital surfactant deficiency. Ultrastruct Pathol 29: 503-509. doi:10.1080/01913120500323480

El Boustany P, Epaud R, Grosse C, Barriere F, Grimont-Rolland E, Carsin A, Dubus JC. 2018. Unusual long survival despite severe lung disease of a child with biallelic loss of function mutations in ABCA-3. Respir Med Case Rep 23: 173-175. doi:10.1016/j.rmcr.2018.03.004

Fitzgerald ML, Xavier R, Haley KJ, Welti R, Goss JL, Brown CE, Zhuang DZ, Bell SA, Lu N, McKee M, et al. 2007. $A B C A 3$ inactivation in mice causes respiratory failure, loss of pulmonary surfactant, and depletion of lung phosphatidylglycerol. J Lipid Res 48: 621-632. doi:10.1194/jlr.M600449-JLR200

Garmany TH, Moxley MA, White FV, Dean M, Hull WM, Whitsett JA, Nogee LM, Hamvas A. 2006. Surfactant composition and function in patients with ABCA3 mutations. Pediatr Res 59: 801-805. doi:10.1203/01.pdr $.0000219311 .14291 . d f$

Hammel M, Michel G, Hoefer C, Klaften M, Müller-Höcker J, de Angelis MH, Holzinger A. 2007. Targeted inactivation of the murine $A b c a 3$ gene leads to respiratory failure in newborns with defective lamellar bodies. Biochem Biophys Res Commun 359: 947-951. doi:10.1016/j.bbrc.2007.05.219

Hartman P, Beckman K, Silverstein K, Yohe S, Schomaker M, Henzler C, Onsongo G, Lam HC, Munro S, Daniel $\mathrm{J}$, et al. 2019. Next generation sequencing for clinical diagnostics: five year experience of an academic laboratory. Mol Genet Metab Rep 19: 100464. doi:10.1016/j.ymgmr.2019.100464

Karczewski KJ, Francioli LC, Tiao G, Cummings BB, Alföldi J, Wang Q, Collins RL, Laricchia KM, Ganna A, Birnbaum DP, et al. 2019. Variation across 141,456 human exomes and genomes reveals the spectrum of loss-of-function intolerance across human protein-coding genes. bioRxiv doi:10.1101/531210

Matsumura Y, Ban N, Ueda K, Inagaki N. 2006. Characterization and classification of ATP-binding cassette transporter ABCA3 mutants in fatal surfactant deficiency. J Biol Chem 281: 34503-34514. doi:10.1074/ jbc.M600071200

Meng L, Pammi M, Saronwala A, Magoulas P, Ghazi AR, Vetrini F, Zhang J, He W, Dharmadhikari AV, Qu C, et al. 2017. Use of exome sequencing for infants in intensive care units: ascertainment of severe singlegene disorders and effect on medical management. JAMA Pediatr 171: e173438. doi:10.1001/jamapedi atrics.2017.3438

Nogee LM, Wert SE, Proffit SA, Hull WM, Whitsett JA. 2000. Allelic heterogeneity in hereditary surfactant protein B (SP-B) deficiency. Am J Respir Crit Care Med 161: 973-981. doi:10.1164/ajrccm.161.3.9903153

Paolini A, Baldassarre A, Del Gaudio I, Masotti A. 2015. Structural features of the ATP-binding cassette (ABC) transporter ABCA3. Int J Mol Sci 16: 19631-19644. doi:10.3390/ijms160819631

Peca D, Cutrera R, Masotti A, Boldrini R, Danhaive O. 2015. ABCA3, a key player in neonatal respiratory transition and genetic disorders of the surfactant system. Biochem Soc Trans 43: 913-919. doi:10.1042/ BST20150100 
Perez-Gil J, Weaver TE. 2010. Pulmonary surfactant pathophysiology: current models and open questions. Physiology (Bethesda) 25: 132-141. doi:10.1152/physiol.00006.2010

Richards S, Aziz N, Bale S, Bick D, Das S, Gastier-Foster J, Grody WW, Hegde M, Lyon E, Spector E, et al. 2015 Standards and guidelines for the interpretation of sequence variants: a joint consensus recommendation of the American College of Medical Genetics and Genomics and the Association for Molecular Pathology. Genet Med 17: 405-424. doi:10.1038/gim.2015.30

Schindlbeck U, Wittmann T, Hoppner S, Kinting S, Liebisch G, Hegermann J, Griese M. 2018. ABCA3 missense mutations causing surfactant dysfunction disorders have distinct cellular phenotypes. Hum Mutat 39: 841850. doi:10.1002/humu.23416

Shulenin S, Nogee LM, Annilo T, Wert SE, Whitsett JA, Dean M. 2004. ABCA3 gene mutations in newborns with fatal surfactant deficiency. N Engl J Med 350: 1296-1303. doi:10.1056/NEJMoa032178

Wambach JA, Casey AM, Fishman MP, Wegner DJ, Wert SE, Cole FS, Hamvas A, Nogee LM. 2014a. Genotype-phenotype correlations for infants and children with ABCA3 deficiency. Am J Respir Crit Care Med 189: 1538-1543. doi:10.1164/rccm.201402-0342OC

Wambach JA, Wegner DJ, Heins HB, Druley TE, Mitra RD, Hamvas A, Cole FS. 2014b. Synonymous ABCA3 variants do not increase risk for neonatal respiratory distress syndrome. J Pediatr 164: 1316-1321. e1313. doi:10.1016/j.jpeds.2014.02.021

Yang R, Nelson AC, Henzler C, Thyagarajan B, Silverstein KA. 2015. Scanlndel: a hybrid framework for indel detection via gapped alignment, split reads and de novo assembly. Genome Med 7: 127. doi:10.1186/ s13073-015-0251-2 


\section{COLD SPRING HARBOR Molecular Case Studies}

\section{Neonatal respiratory failure due to novel compound heterozygous mutations in the ABCA3 lipid transporter}

Zoltán N. Oltvai, Eric A. Smith, Katie Wiens, et al.

Cold Spring Harb Mol Case Stud 2020, 6: a005074

Access the most recent version at doi: $10.1101 / \mathrm{mcs} . \mathrm{a} 005074$

\section{Supplementary http://molecularcasestudies.cshlp.org/content/suppl/2020/06/12/mcs.a005074.D Material C1}

References This article cites 26 articles, 8 of which can be accessed free at: http://molecularcasestudies.cshlp.org/content/6/3/a005074.full.html\#ref-list-1

License This article is distributed under the terms of the Creative Commons Attribution-NonCommercial License, which permits reuse and redistribution, except for commercial purposes, provided that the original author and source are credited.

Email Alerting Receive free email alerts when new articles cite this article - sign up in the box at the Service top right corner of the article or click here. 